\title{
Characterization of limb lymphedema using the statistical analysis of ultrasound backscattering
}

\author{
Ya-Lun Lee,2\#, Yen-Ling Huang ${ }^{1 \#}$, Sung-Yu Chu ${ }^{1}$, Wen-Hui Chan ${ }^{1}$, Ming-Huei Cheng ${ }^{3}$, Ying-Hsiu Lin ${ }^{4}$, \\ Tu-Yung Chang ${ }^{5}$, Chih-Kuang Yeh ${ }^{2}$, Po-Hsiang Tsui ${ }^{1,4,6}$ \\ ${ }^{1}$ Department of Medical Imaging and Intervention, Chang Gung Memorial Hospital at Linkou, Taoyuan, Taiwan; ${ }^{2}$ Department of Biomedical \\ Engineering and Environmental Sciences, National Tsing Hua University, Hsinchu, Taiwan; ${ }^{3}$ Division of Reconstructive Microsurgery, Department \\ of Plastic and Reconstructive Surgery, Chang Gung Memorial Hospital, Chang Gung University, College of Medicine, Taoyuan, Taiwan; \\ ${ }^{4}$ Department of Medical Imaging and Radiological Sciences, College of Medicine, Chang Gung University, Taoyuan, Taiwan; ${ }^{5}$ Department of Public \\ Health, College of Public Health, China Medical University, Taichung, Taiwan; ${ }^{6}$ Institute for Radiological Research, Chang Gung University and \\ Chang Gung Memorial Hospital at Linkou, Taoyuan, Taiwan
}

\#These authors contributed equally to this work.

Correspondence to: Prof. Po-Hsiang Tsui, PhD. Department of Medical Imaging and Radiological Sciences, College of Medicine, Chang Gung University, Taoyuan, Taiwan. Email: tsuiph@mail.cgu.edu.tw; Sung-Yu Chu, MD. Department of Medical Imaging and Intervention, Chang Gung Memorial Hospital at Linkou, Taoyuan, Taiwan. Email: sungyu.chu@msa.hinet.net.

Background: Lymphedema is a disease in which tissue swelling is caused by interstitial fluid retention in subcutaneous tissue. It is caused by a compromised lymphatic system. Lymphoscintigraphy is the current and primary modality used to assess lymphatic system dysfunction. Ultrasound elastography is a complementary tool used for evaluating the tissue stiffness of the lymphedematous limb. Tissue stiffness implies the existence of changes in tissue microstructures. However, ultrasound features related to tissue microstructures are neglected in clinical assessments of lymphedematous limbs. In this study, we aimed to evaluate the lymphedematous diagnostic values of ultrasound Nakagami and entropy imaging, which are, respectively, model- and nonmodel-based backscattered statistical analysis methods for scatterer characterization.

Methods: A total of 60 patients were recruited, and lymphoscintigraphy was used to score the patient's clinical severity of each of their limb lymphedema (0: normal; 1: partial lymphatic obstruction; and 2: total lymphatic obstruction). We performed ultrasound examinations to acquire ultrasound backscattered signals for B-mode, Nakagami, and entropy imaging. The envelope amplitude, Nakagami, and entropy values, as a function of the patients' lymphatic obstruction grades, were expressed in terms of their median and interquartile range (IQR). The values were then used in both an independent $t$ test and a receiver operating characteristic (ROC) curve analysis.

Results: For each increase in a patient's score from 0 to 2, the envelope amplitude values were 405.44 (IQR: 238.72-488.17), 411.52 (IQR: 298.53-644.25), and 476.37 (IQR: 348.86-648.16), respectively. The Nakagami parameters were 0.16 (IQR: 0.14-0.22), 0.26 (IQR: 0.23-0.34), and 0.24 (IQR: 0.16-0.36), respectively, and the entropy values were 4.55 (IQR: 4.41-4.66), 4.86 (IQR: 4.78-4.99), and 4.87 (IQR: 4.81-4.97), respectively. The $\mathrm{P}$ values between the normal control and lymphedema groups obtained from B-mode and Nakagami analysis were larger than 0.05; whereas that of entropy imaging was smaller than 0.05 . The areas under the ROC curve for B-mode, Nakagami, and entropy imaging were 0.64 (sensitivity: $70 \%$; specificity: 47.5\%), 0.75 (sensitivity: 70\%; specificity: 75\%), and 0.94 (sensitivity: 95\%; specificity: $87.5 \%$ ), respectively.

Conclusions: The current findings demonstrated the diagnostic values of ultrasound Nakagami and entropy imaging techniques. In particular, the use of non-model-based entropy imaging enables for improved performance when characterizing limb lymphedema. 
Keywords: Lymphedema; ultrasound imaging; Nakagami distribution; information entropy

Submitted Jun 30, 2019. Accepted for publication Oct 08, 2019.

doi: 10.21037/qims.2019.10.12

View this article at: http://dx.doi.org/10.21037/qims.2019.10.12

\section{Introduction}

Lymphedema is an illness that arises from the impairment of the lymphatic drainage system. It can be caused by certain congenital and acquired reasons (1). Secondary lymphedema in the upper and lower limbs is frequently seen in cancer patients (2). Lymphedema causes swelling in the affected region because of the accumulation of proteinrich interstitial fluid in subcutaneous tissue. For patients with chronic lymphedema, both physical disabilities and psychological stress generally increase with the progression of the disease (3). To reduce patient discomfort and improve quality of life, an accurate diagnosis of lymphedema is essential for prognosis and treatment planning.

In general, the clinical severity of lymphedema can be classified by performing physical examinations that evaluate both the condition of swelling in the limb and the edema pitting scale. Several medical imaging modalities have also been applied to diagnose lymphedema; for example, $\mathrm{X}$-ray lymphography, lymphoscintigraphy, computed tomography, magnetic resonance imaging, near-infrared fluorescence imaging, and ultrasound (4). Among these, lymphoscintigraphy is the primary imaging modality used to assess lymphatic system dysfunction; it has been considered the criterion standard for decades (5). However, lymphoscintigraphy cannot provide quantitative clues for measuring changes in the stiffness of the lymphedematous limb. Such stiffness is a major cause of discomfort and impaired limb function in patients. To address the deficiencies of lymphoscintigraphy, some studies have proposed using ultrasound elastography to evaluate the tissue stiffness of the lymphedematous limb. It has been shown that the measure of semi-quantitative ultrasound strain elastography correlates with indocyanine green lymphography $(6,7)$. Recent studies have further used acoustic radiation force impulse (ARFI) elastography to perform quantitative measures of the shear-wave velocity. They do so as an evaluation of stiffness. This indicates that the stiffness of cutaneous and subcutaneous tissues in lymphedematous limbs is significantly higher than that of unaffected sides (8). In particular, ARFI elastography can be used to support the treatment planning of pneumatic compression for postmastectomy lymphedema (3).

In principle, stiffness increase in lymphedematous limbs implies the existence of changes in internal microstructures. This is supported by a previous study, demonstrating that chronic lymphedema induces complications such as inflammation, adipose tissue hypertrophy, fibrosis, and recurrent infections (9). This finding indicates that information associated with changes in tissue microstructures are complementary clues and should thus be considered in the ultrasound characterization of lymphedema. Generally, a biological tissue can be modeled as a scattering medium consisting of numerous scatterers. These scatterers interact with the incident ultrasound to contribute backscattered signals (10). The variety of scatterer arrangements results in various behavior of both the ultrasound wave interference and the corresponding backscattered signals. In the literature, several statistical models have been used to analyze the statistics of backscattered signals and characterize tissue microstructures. Of these, the Nakagami distribution, given its simplicity and low computational complexity, is the most frequently adopted approximation model $(11,12)$. Besides these model-based approaches, information entropy has been proposed as a non-model-based method to estimate backscattered statistics to describe microstructural changes $(13,14)$. Compared with the statistical distribution models, the entropy approach allows one to use any type of data (regardless of its distribution) to estimate the statistical properties of backscattered signals (signal uncertainty) $(15-17)$

In the imaging of lymphedematous limbs, the diagnostic value of the model- and non-model-based backscattered methods of statistical analysis remains unknown. Thus, in this study, we aimed to explore the performance of ultrasound Nakagami and entropy parametric imaging for characterizing lymphedema. In subsequent sections, we describe how we recruited patients with lymphedema for the acquisition and analysis of our clinical data. Our results indicate that compared with the model-based Nakagami imaging, ultrasound entropy imaging achieves superior diagnostic accuracy with regards to limb lymphedema. 


\section{Methods}

\section{Participants}

This study was approved by the Institutional Review Board at Chang Gung Memorial Hospital in Linkou, Taiwan. A total of 60 patients were recruited from the Department of Medical Imaging and Intervention; they all signed informed consent forms. All participants underwent lymphoscintigraphy to confirm the diagnosis of unilateral lower limb lymphedema. For each subject, the clinical severity of limb lymphedema was scored using the new Taiwan lymphoscintigraphy staging system (0: normal; 1: partial lymphatic obstruction; 2: total lymphatic obstruction) (18). Characteristics of the participants, namely age, sex, and body mass index (BMI), were recorded.

\section{Ultrasound data acquisition and analysis}

After performing lymphoscintigraphy, all patients underwent an ultrasound screening on bilateral lower limbs (at the medial surface of the leg $10 \mathrm{~cm}$ above the medial malleolus). The screening was conducted by a radiologist who was blinded to the lymphoscintigraphy reports. A clinical ultrasound scanner (ACUSON S3000, Siemens, Mountain View, CA, USA) was equipped with a linear array transducer with a $7.5 \mathrm{MHz}$ central frequency (Model 9L4). It was used to acquire raw image data, consisting of 912 A-lines of backscattered radiofrequency (RF) signals. The pulse length of the transducer was approximately $1 \mathrm{~mm}$, and the sampling rate was $40 \mathrm{MHz}$.

For each piece of raw data, the envelope image was obtained by taking the absolute value of the Hilbert transform of the backscattered RF signals. The B-mode image was constructed using the logarithm-compressed envelope image at a dynamic range of $40 \mathrm{~dB}$. The uncompressed envelope image was concurrently processed using the sliding window technique to generate both ultrasound Nakagami and entropy parametric images. Details regarding the algorithmic scheme and the mathematical formula for estimating both the Nakagami and entropy values can be found in a previous study (16). For each window location, the Nakagami parameter $m$ was estimated using the moment-based estimator, as given by

$$
m=\frac{\left[E\left(A^{2}\right)\right]^{2}}{E\left[A^{2}-E\left(A^{2}\right)\right]^{2}}
$$

where $E($.$) is the statistical mean operator, and A$ means the local data acquired by the window. The entropy value was calculated using the statistical histogram of backscattered envelope data $w(y)$ (number of bins $=100$ ) in Eq. [2].

$$
H_{c} \equiv-\int_{y_{\min }}^{y_{\max }} w(y) \log _{2}[w(y)] d y
$$

where $y_{\min }$ and $y_{\max }$ represent the minimal and maximal values of the local data in the window. The window side length for constructing a Nakagami image was set at three times the pulse length of the transducer. For entropy imaging, we adopted one pulse length as the window side length. This is because entropy imaging allows for small-window parametric imaging $(15,16)$. A window overlap ratio of $50 \%$ was used to achieve a compromise between image resolution and computational time. To quantitatively evaluate changes in the tissue microstructure with limb lymphedema, a region of interest (ROI), manually outlined on the B-mode image, was applied to the corresponding Nakagami and entropy images to calculate the average of the image pixel values within the ROI, which was empirically shown as an appropriate index for tissue quantitative analysis (15-17).

It can be expected that several factors associated with the ROI selection affect the results, including the ROI location, artifacts influence, and information contents; however, no standard criteria for determining the ROI on the images of lymphedema are currently available. To reduce the measurement bias in characterizing lymphedema, we established the following rules for determining the ROI: (I) the ROI location is placed on the subcutaneous layer, which is anatomically located between the cutaneous and muscular layers, for data analysis, (II) blurry boundaries caused by reverberation artifacts are excluded, and (III) the ROI should be as large as possible to provide sufficient backscattering information.

\section{Statistical analysis}

The envelope amplitude, Nakagami, and entropy values, as a function of the lymphatic obstruction grades, were expressed in terms of the median and interquartile range (IQR). The independent $t$ test was used to compare the data between each group $(\mathrm{P}<0.05$ was considered statistically significant). We also used a receiver operating characteristic (ROC) curve analysis with a 95\% confidence interval (CI) to evaluate the diagnostic performance of the Nakagami and entropy imaging. The area under the ROC (AUROC), accuracy, sensitivity, and specificity were determined. All statistical analyses were performed using SigmaPlot 
Table 1 Characteristics of participants with lower limb lymphedema

\begin{tabular}{lc}
\hline Characteristics & Result \\
\hline Male/female & $6 / 54$ \\
Age, years & \\
Mean \pm standard deviation [range] & $59 \pm 11.7[29-85]$ \\
Median & 61 \\
BMl, kg/m ${ }^{2}$ & \\
Mean \pm standard deviation (range) & $25 \pm 4.4(17-37.9)$ \\
Median & 24.8 \\
Lymphatic obstruction grade ${ }^{*}$ & \\
0 & 20 \\
1 & 20 \\
2 & 20 \\
Cause of lymphedema & \\
Primary & 64 \\
Secondary & \\
\hline
\end{tabular}

Unless otherwise noted, data are numbers of patients. ${ }^{\star} T h e$ lymphatic obstruction grade on both affected and contralateral limbs were assessed by the novel Taiwan Lymphoscintigraphy Staging System (18). BMI, body mass index.

software (Version 12.0, Systat Software, Inc., San Jose, CA, USA).

\section{Results}

The characteristics of participants with unilateral lower limb lymphedema are summarized in Table 1. The ultrasound B-mode, Nakagami, and entropy images for each stage of lymphatic obstruction are depicted in Figure 1. The brightness of these images increased with the increase in the severity of limb lymphedema. This indicates that during the formation of lymphedema, the image intensity varied, and the ultrasound model- and non-model-based statistical parametric images enable visualizing the changes in the backscattered statistics. Note that the colors appearing on the parametric images were assigned according to the pixel strength for the purpose of visualization only. The features (or textures) of an ultrasound parametric image should be quantified based on the statistical descriptions of the parameter values. For this reason, the user-defined pseudo color is not recommended as the feature to characterize lymphedema. As mentioned in Materials and Methods, the average of the parameter values in the ROI is a workable index for tissue quantitative analysis.

The envelope amplitude, Nakagami, and entropy values corresponding to each lymphatic obstruction grade are presented in Figure 2. With every increase of the score from 0 to 2, the envelope amplitude values were 405.44 (IQR: 238.72-488.17), 411.52 (IQR: 298.53-644.25), and 476.37 (IQR: 348.86-648.16), respectively; the Nakagami parameters were 0.16 (IQR: $0.14-0.22$ ), 0.26 (IQR: $0.23-0.34$ ), and 0.24 (IQR: $0.16-0.36$ ), respectively; and the entropy values were 4.55 (IQR: 4.41-4.66), 4.86 (IQR: 4.78-4.99), and 4.87 (IQR: 4.81-4.97), respectively. Compared with the B-mode and Nakagami values, the entropy parameter had statistically significant difference between normal and partial or total lymphatic obstructions $(\mathrm{P}<0.05)$. The $\mathrm{P}$ value between the normal control and lymphedema groups obtained from the entropy analysis was also smaller than 0.05 . According to the results displayed in Figure 3, ultrasound entropy imaging seemed to provide an improved characterization of limb lymphedema. The AUROC was 0.64 (sensitivity: 70\%; specificity: $47.5 \%$ ) for B-mode imaging, 0.75 (sensitivity: 70\%; specificity: $75 \%$ ) for Nakagami imaging, and 0.94 (sensitivity: 95\%; specificity: $87.5 \%$ ) for ultrasound entropy imaging. Evidently, conventional B-mode imaging is unable to characterize limb lymphedema. However, the current findings confirmed the diagnostic value of ultrasound Nakagami and entropy imaging techniques in grading limb lymphedema. Specifically, non-model-based entropy imaging achieves superior performance. Performance comparisons between ultrasound B-mode, Nakagami, and entropy imaging are summarized in Table 2.

\section{Discussion}

\section{Significance of this study}

In this study, we evaluated the use of ultrasound Nakagami and entropy imaging for staging lymphedema. The literature has yet to pay attention to the analysis of ultrasound backscattered statistics for lymphedematous tissues. However, the current findings in this study support the value of using ultrasound backscattering analysis for diagnosing lymphedema. Specifically, compared with ultrasound parametric imaging based on the Nakagami statistical model, the information entropy approach achieves significantly better performance in the diagnosis and staging of limb lymphedema. This demonstrates that 

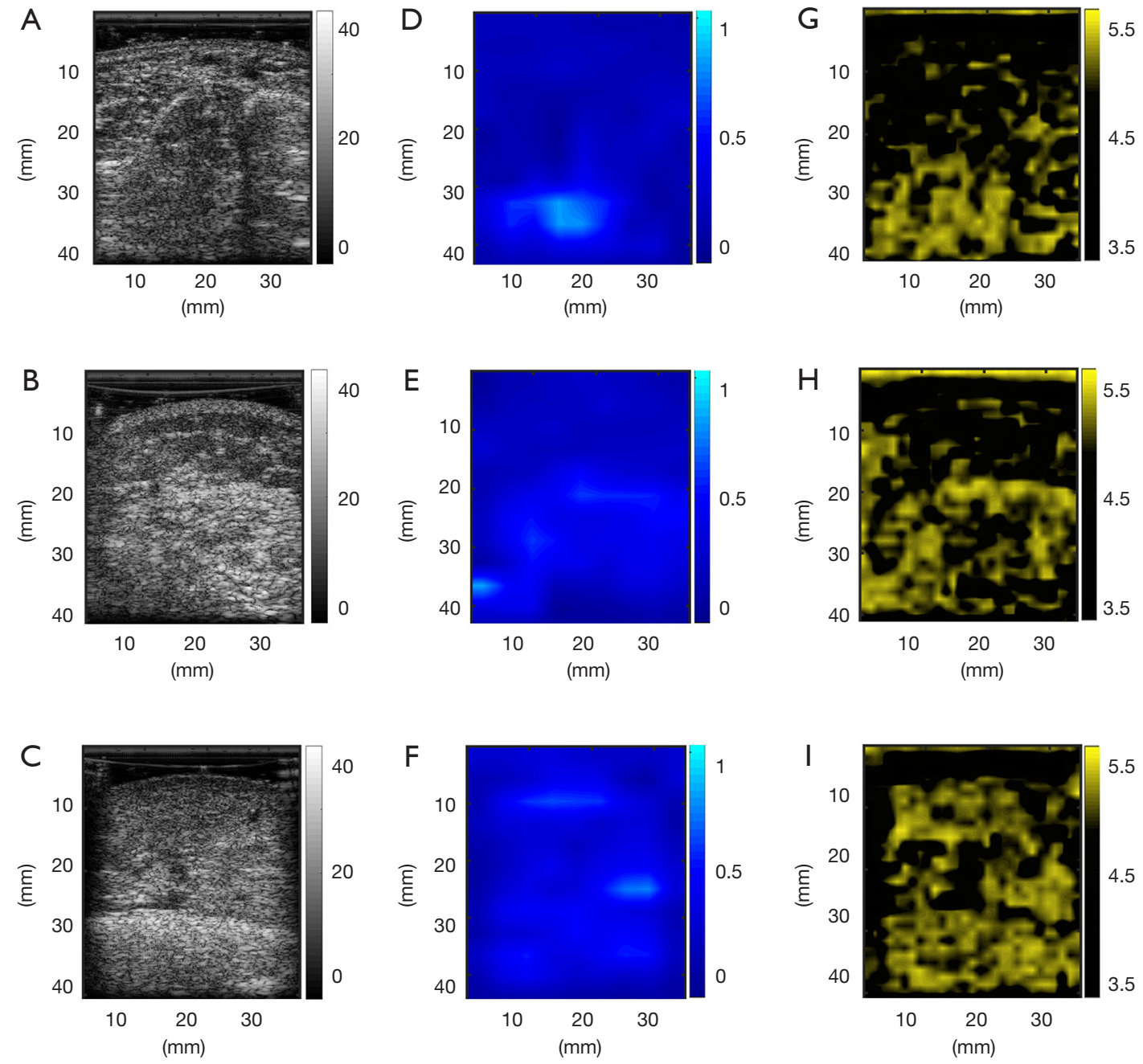

Figure 1 Ultrasound parametric imaging for each stage of lymphatic obstruction (top: grade 0; middle: grade 1; bottom: grade 2). Images (A) to $(\mathrm{C})$ are the B-mode images, (D) to $(\mathrm{F})$ are the Nakagami images, and $(\mathrm{G})$ to $(\mathrm{I})$ are the entropy images. The brightness of the Nakagami and entropy images increased with each increase of the severity of limb lymphedema, indicating that ultrasound model- and non-modelbased statistical parametric images enable the visualization of changes in the backscattered statistics during the formation of lymphedema.

the information uncertainty of ultrasound backscattered signals is a means of characterizing microstructures that is able to improve the clinical assessment of lymphedema. To the best of our knowledge, this study is the first to reveal the usefulness of ultrasound backscattered statistics in the clinical evaluation of lymphedema.

\section{The relationship between changes in microstructures and backscattered statistics}

According to the results, ultrasound Nakagami and entropy values increase with the severity of lymphedema.
Physically, this means that the backscattered statistics of advanced stages of lymphedema are closer to the Rayleigh distribution. The signal uncertainty (or complexity) of ultrasound backscattering is also enhanced, corresponding to higher entropy values. A primary mechanism that causes increases in the Nakagami and entropy values could be histological changes in lymphedema. In lymphedematous limbs, stagnation of lymph fluid circulation gradually triggers changes in tissue microstructures such as adipose tissues and fibrosis deposition (19). It should be noted that the fat cell also belongs to both an endocrine organ and a cytokine-activated cell (20). During chronic inflammation, 
A

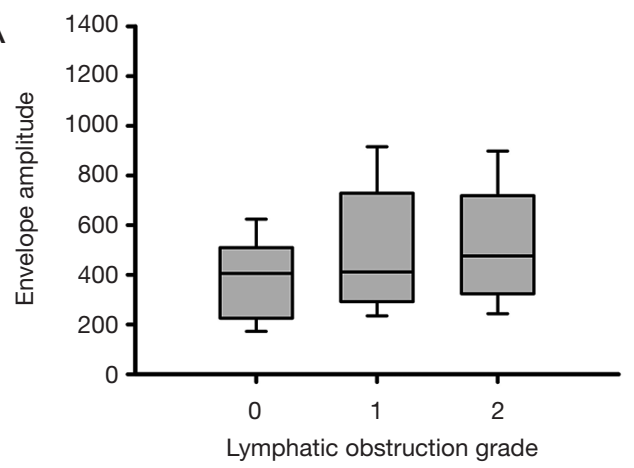

B
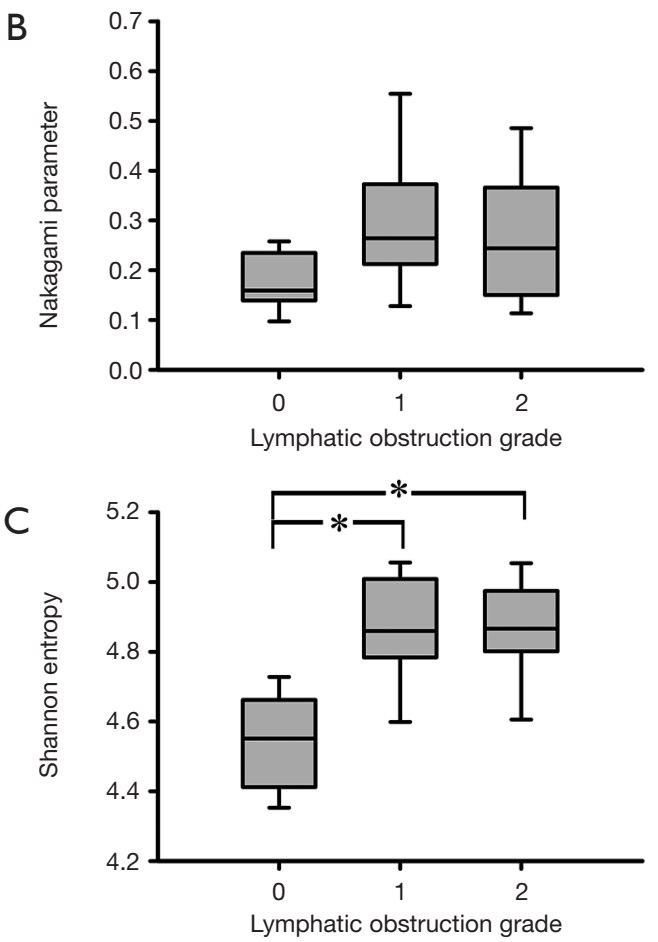

$\mathrm{D}$

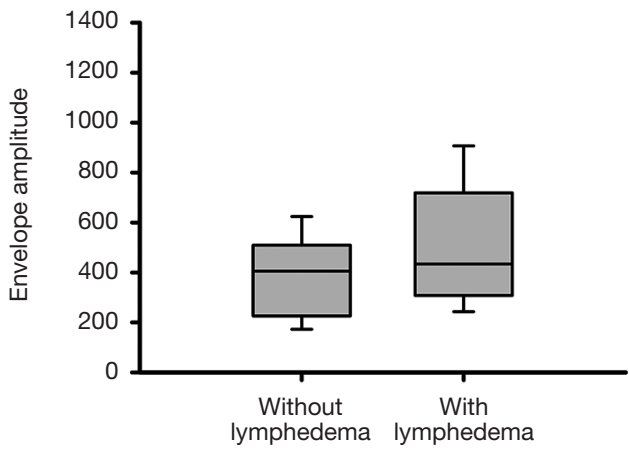

$\mathrm{E}$

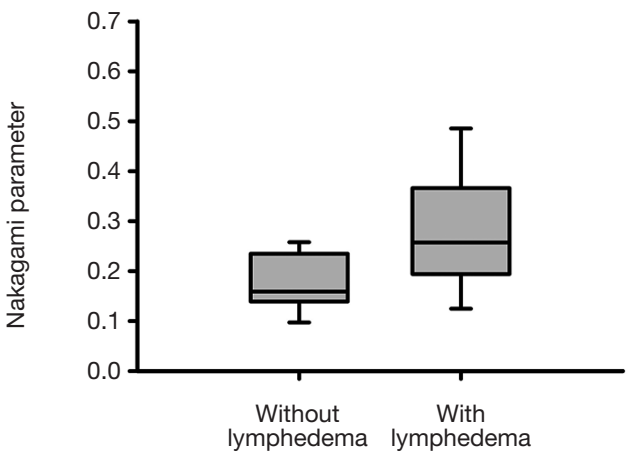

$\mathrm{F}$

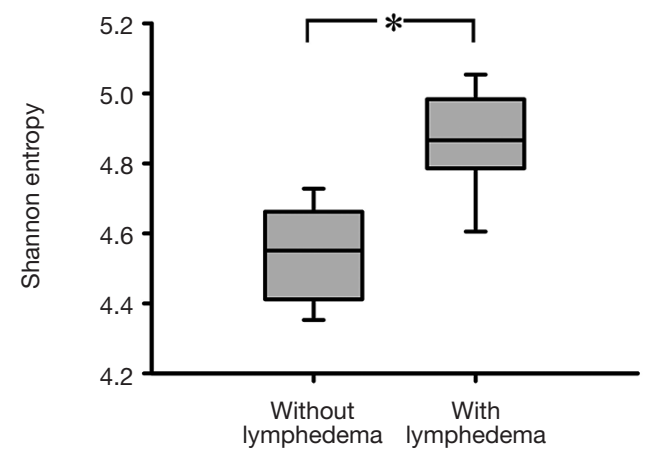

Figure 2 Statistical analysis and comparisons for B-mode and parametric imaging of limb lymphedema. (A) to (C) B-mode, Nakagami, and entropy values corresponding to each lymphatic obstruction grade; (D) to (F) comparisons of the values obtained without and with lymphedema. The symbol (*) means $\mathrm{P}<0.05$. Envelope amplitude, Nakagami, and entropy values increased with each increase of lymphedema severity. The entropy parameter had statistically significant difference between normal and partial or total lymphatic obstructions. The difference between the normal control and lymphedema groups obtained from the entropy analysis was also statistically significant.

excess adipose tissues to a slow lymph flow, and adipocytes transformed from macrophages accelerate lipogenesis and fat deposition (21). Thereafter, because of pinocytosis of the white blood cells and activation of fibroblasts, the connective tissues increase, hardening subcutaneous lymphedematous tissues $(22,23)$. The aforementioned changes in histological features imply an increase in the number of scatterers in the resolution cell of the transducer, thereby strengthening the effect of constructive wave interference. As demonstrated in previous studies, this phenomenon reduces the difference between the backscattered statistics and the Rayleigh distribution. It also enlarges the uncertainty of ultrasound backscattered signals $(16,24,25)$.

\section{Comparison between the statistical model and information entropy}

Our results indicate that the statistical model's accuracy is 


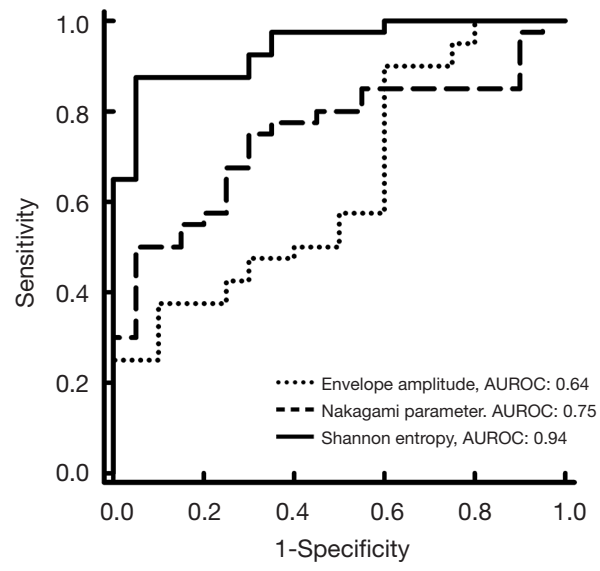

Figure 3 Receiver operating characteristic (ROC) curves obtained from ultrasound B-mode, Nakagami, and entropy imaging. The area under the ROC (AUROC) for B-mode imaging was 0.64 (sensitivity: $70 \%$; specificity: $47.5 \%$ ), and that for Nakagami parametric imaging was 0.75 (sensitivity: 70\%; specificity: 75\%). Compared with the B-mode envelope amplitude and Nakagami parameter, information entropy enables to stage lymphedematous limbs with a significantly improved diagnostic accuracy (the AUROC: 0.94; sensitivity: 95\%; specificity: $87.5 \%$ ).

inferior to information entropy's for characterizing limb lymphedema. This suggests that information entropy has a diagnostic advantage in imaging lymphedema. One possible reason why Nakagami imaging performed worse is that it cannot be applied in the description of the backscattered statistics of lymphedematous limbs. A previous study determined that because of the existence of complex structures in the tissue background, Nakagami parameter estimation is susceptible to errors and incoherent results (26). Fundamentally, lymphedematous tissue is not a homogeneous scattering medium. The existence of structural information is the likely cause of the Nakagami parameter's reduced diagnostic accuracy. Compared with the statistical model, information entropy was defined mathematically as informational content. In practical applications, information entropy can reveal the signal's relative information quantity. This provides clues pertaining to tissue properties regardless of data distribution. We cannot deny the usefulness of the statistical model in tissue characterization, but with respect to the diagnosis of lymphedema, the use of statistical models needs to be further refined. Prior to such refinements, information entropy can be used as an appropriate quantitative approach for lymphedema detection.
Table 2 Diagnostic performance of ultrasound B-mode (using envelope amplitude) and parametric imaging (using Nakagami distribution and information entropy) for the assessment of limb lymphedema

\begin{tabular}{lccc}
\hline Parameter & $\begin{array}{c}\text { Envelope } \\
\text { amplitude }\end{array}$ & $\begin{array}{c}\text { Nakagami } \\
\text { parameter }\end{array}$ & $\begin{array}{c}\text { Shannon } \\
\text { entropy }\end{array}$ \\
\hline Cutoff value & 460.7273 & 0.2004 & 4.7285 \\
Sensitivity, \% & 70 & 70 & 95 \\
Specificity, \% & 47.5 & 75 & 87.5 \\
Accuracy, \% & 55 & 73.33 & 90 \\
LR+ & 1.33 & 2.8 & 7.6 \\
LR- & 0.63 & 0.4 & 0.06 \\
PPV, \% & 40 & 58.33 & 79.17 \\
NPV, \% & 76 & 83.33 & 97.22 \\
AUROC & 0.64 & 0.75 & 0.94 \\
(95\% Cl) & $(0.48-0.79)$ & $(0.62-0.87)$ & $(0.89-0.99)$ \\
\hline
\end{tabular}

LR+, positive likelihood ratio; LR-, negative likelihood ratio; PPV, positive predictive value; NPV, negative predictive value; AUROC, area under the receiver operating characteristic curve; $\mathrm{Cl}$, confidence interval.

\section{Comparisons with ultrasound elastography and the conventional B-scan}

Recall that a previous study has explored using the ARFI to measure lymphedematous limbs, improving clinical diagnosis of lymphedema by offering information on tissue stiffness (8). However, the ARFI technique only allows local measurements, hindering overall evaluations of subcutaneous tissues in lymphedematous limbs. Compared with the ARFI, ultrasound statistical parametric imaging provides information associated with changes in tissue microstructures as additional clues for characterization of lymphedema. The current results showed that the AUROC of ultrasound entropy imaging in diagnosing lymphedema was 0.94 , which is higher than that of the ARFI (0.83) revealed in the previous study (8). Evidently, ultrasound entropy imaging has the potential in the assessment of lymphedema detection.

In clinical settings, conventional ultrasound B-mode imaging is still a convenient and fast screening tool for examinations of lymphedematous limbs; whereas, it is difficult to characterize tissue microstructures for further clinical decision making, as supported by the results in Figure 2. Although a lot of image processing and display 
methods of ultrasound B-scan are available, concluding the best processing approach for the diagnosis of lymphedema is still challenging because the features of ultrasound B-scan are system-dependent, easily affected by user experiences, and vary with the stage of lymphedema. Therefore, prior to using conventional B-scan as a reliable diagnostic tool for lymphedema, systematic investigations and validations are needed in the future. On the contrary, ultrasound statistical parametric imaging using the unprocessed raw RF data provides a more objective evaluation of lymphedema.

\section{Limitations and future suggestions}

Some limitations of this study and suggestions for future directions in research are given in the following. First, the number of patients in our experimental design was limited. Using ultrasound backscattered data acquired from various machines may have also produced bias in parameter estimations. Thus, the standard cutoff values for staging lymphedema using Nakagami and entropy imaging ought to be investigated further. Second, cutaneous tissues were not considered in our measurements. This is because of difficulties obtaining a larger ROI for ultrasound parametric imaging and analysis. In the future, one can consider higher-frequency ultrasound for imaging cutaneous tissues with lymphedema for parametric analysis. We also suggest that prior to using the method of backscattered statistics as a diagnostic tool for limb lymphedema, cross-platform ultrasound systems should be used to conduct large-scale clinical validations.

\section{Conclusions}

In this study, we explored the diagnostic value of using model- and non-model-based backscattered statistical analysis methods (the Nakagami distribution and information entropy) in imaging lymphedematous limbs for the clinical evaluation of disease severity. Conventional B-mode imaging was shown to be unable to characterize limb lymphedema; whereas, the experimental results supported the usefulness of both ultrasound Nakagami and entropy imaging in lymphedema characterization. Compared with the Nakagami parameter, information entropy enables superior diagnostic accuracy in the staging of lymphedematous limbs. This may be attributed to how entropy is still applicable for describing the statistical properties of backscattered signals even if the structural information able to change the data distribution exists. In future applications, ultrasound entropy imaging may be a useful tool for the assessment of limb lymphedema.

\section{Acknowledgments}

Funding: This work was supported by the Ministry of Science and Technology in Taiwan (Grant Nos. MOST 106-2221-E-182-023-MY3 and 108-2218-E-182-001) and the Chang Gung Memorial Hospital at Linkou in Taiwan (Grant Nos. CORPG1G0091 and CMRPD1H0381).

\section{Footnote}

Conflicts of Interest: The authors have no conflicts of interest to declare.

Ethical Statement: The subject gave informed consent. This study was approved by the Institutional Review Board at Chang Gung Memorial Hospital in Linkou, Taiwan.

\section{References}

1. Polat AK, Karabacak U, Mutlu V, Tomak L, Bilgici A. Early diagnosis of lymphedema after breast cancer treatment: bio-impedance spectroscopy. J Breast Health 2017;13:83-87.

2. Shah C, Arthur D, Riutta J, Whitworth P, Vicini FA. Breast-cancer related lymphedema: a review of procedure-specific incidence rates, clinical assessment AIDS, treatment paradigms, and risk reduction. Breast J 2012;18:357-61.

3. Bok SK, Jeon Y, Lee JA, Ahn SY. Evaluation of stiffness in postmastectomy lymphedema using acoustic radiation force impulse imaging: a prospective randomized controlled study for identifying the optimal pneumatic compression pressure to reduce stiffness. Lymphat Res Biol 2018;16:36-42.

4. O’Donnell TF Jr, Rasmussen JC, Sevick-Muraca EM. New diagnostic modalities in the evaluation of lymphedema. J Vasc Surg Venous Lymphat Disord 2017;5:261-73.

5. Munn LL, Padera TP. Imaging the lymphatic system. Microvasc Res 2014;96:55-63.

6. Suehiro K, Nakamura K, Morikage N, Murakami M, Yamashita O, Ueda K, Samura M, Hamano K. Real-time tissue elastography assessment of skin and subcutaneous tissue strains in legs with lymphedema. J Med Ultrason (2001) 2014;41:359-64.

7. Hayashi N, Yamamoto T, Hayashi A, Yoshimatsu H. 
Correlation between indocyanine green (ICG) patterns and real-time elastography images in lower extremity lymphedema patients. J Plast Reconstr Aesthet Surg 2015;68:1592-99.

8. Chan WH, Huang YL, Lin C, Lin CY, Cheng MH, Chu SY. Acoustic radiation force impulse elastography: tissue stiffness measurement in limb lymphedema. Radiology 2018;289:759-65.

9. Ly CL, Kataru RP, Mehrara BJ. Inflammatory manifestations of lymphedema. Int J Mol Sci 2017;18:171.

10. Shung KK, Thieme GA. Ultrasonic scattering in biological tissues, CRC Press, Florida, 1993.

11. Mamou J, Oelze ML. Quantitative ultrasound in soft tissues, Springer, New York, 2013.

12. Tsui PH, Zhou Z, Lin YH, Hung CM, Chung SJ, Wan YL. Effect of ultrasound frequency on the Nakagami statistics of human liver tissues. PLoS One 2017;12:e0181789.

13. Hughes MS. Analysis of digitized waveforms using Shannon entropy. J Acoust Soc Am 1993;93:892-906.

14. Hughes MS, McCarthy JE, Marsh JN, Arbeit JM, Neumann RG, Fuhrhop RW, Wallace KD, Znidersic DR, Maurizi BN, Baldwin SL, Lanza GM, Wickline SA. Properties of an entropy-based signal receiver with an application to ultrasonic molecular imaging. J Acoust Soc Am 2007;121:3542-57.

15. Tsui PH, Chen CK, Kuo WH, Chang KJ, Fang J, Ma HY, Chou D. Small-window parametric imaging based on information entropy for ultrasound tissue characterization. Sci Rep 2017;7:41004.

16. Zhou Z, Tai DI, Wan YL, Tseng JH, Lin YR, Wu S, Yang KC, Liao YY, Yeh CK, Tsui PH. Hepatic steatosis assessment with ultrasound small-window entropy imaging. Ultrasound Med Biol 2018;44:1327-40.

Cite this article as: Lee YL, Huang YL, Chu SY, Chan WH, Cheng MH, Lin YH, Chang TY, Yeh CK, Tsui PH. Characterization of limb lymphedema using the statistical analysis of ultrasound backscattering. Quant Imaging Med Surg 2020;10(1):48-56. doi: 10.21037/qims.2019.10.12
17. Lin YH, Liao YY, Yeh CK, Yang KC, Tsui PH. Ultrasound entropy imaging of nonalcoholic fatty liver disease: association with metabolic syndrome. Entropy 2018;20:893.

18. Cheng MH, Pappalardo M, Lin C, Kuo CF, Lin CY, Chung KC. Validity of the novel Taiwan lymphoscintigraphy staging and correlation of Cheng lymphedema grading for unilateral extremity lymphedema. Ann Surg 2018;268:513-25.

19. Hoffner M, Peterson P, Månsson S, Brorson H. Lymphedema leads to fat deposition in muscle and decreased muscle/water volume after liposuction: a magnetic resonance imaging study. Lymphat Res Biol 2018;16:174-81.

20. Brorson H. Liposuction in lymphedema treatment. J Reconstr Microsurg 2016;32:56-65.

21. Larsen OA, Lassen NA, Quaade F. Blood flow through human adipose tissue determined with radioactive xenon. Acta Physiol Scand 1966;66:337-45.

22. Casley-Smith JR, Gaffney RM. Excess plasma proteins as a cause of chronic inflammation and lymphodema: quantitative electron microscopy. J Pathol 1981;133:243-72.

23. Gaffney RM, Casley-Smith JR. Excess plasma proteins as a cause of chronic inflammation and lymphoedema: biochemical estimations. J Pathol 1981;133:229-42.

24. Tsui PH. Ultrasound detection of scatterer concentration by weighted entropy. Entropy 2015;17:6598-6616.

25. Tsui PH, Wan YL. Effects of fatty infiltration of the liver on the Shannon entropy of ultrasound backscattered signals. Entropy 2016;18:341.

26. Larrue A, Noble JA. Modeling of errors in Nakagami imaging: illustration on breast mass characterization. Ultrasound Med Biol 2014;40:917-30. 